\title{
Zur Kenntnis der Veränderungen des Bulbus durch Druck eines 0rbitaltumors.
}

\author{
Von \\ Prof. Dr. A. Birch-Hirschfeld und Dr. C. Siegfried.
}

Mit 3 Figuren im Text.

Die Art, wie eine Erkrankung der Orbita den Augapfel und den Sehnerven in Mitleidenschaft ziehen und vorübergehenden oder dauernden teilweisen oder völligen Verlust der Sehschärfe veranlassen kann, kann eine recht mannigfaltige sein.

Zunächst kann die Erkrankung auf das Auge und den Opticus selbst übergreifen oder eine Zirkulationsstörung im Bereich der Zentralgefässe zur Folge haben. Durch mechanische Einwirkung auf den Sehnerven kann, wie z. B. die anatomischen Untersuchungen des einen von uns gezeigt haben, nicht nur Stauungspapille bewirkt, sondern eine direkte Faserdegeneration hervorgerufen werden.

Aber auch der Exophthalmus als solcher kann bei höheren Graden den Augapfel gefährden, insofern er ihn des Schutzes der Lider beraubt, was zu entzündlichen Veränderungen der Hornhaut mit ihren oft schweren Folgezuständen (Ulzeration, Perforation) führen kann.

Dass auch der Druck eines Orbitaltumors auf den Bulbus eine Änderung des Brechungszustandes hervorrufen kann, ist eine Tatsache, die seit langem bekannt, vor kurzem erst von $\mathrm{Hand}$ mann hervorgehoben worden ist.

Nach den Erfahrungen an dem reichhaltigen Material der Leipziger Klinik ist diese Refraktionsänderung eine recht häufige Erscheinung bei allen Prozessen, die mit hochgradigem Exophthalmus einhergehen, besonders bei Orbitalgeschwülsten. Meist handelt es sich um einen Astigmatismus, seltener um einfache Myopie oder Hyperopie. Die Art der Refraktionsänderung erklärt 
sich aus der Richtung, in welcher der Druck auf den Augapfel einwirkt, und auch ihr Grad dürfte im allgemeinen der Intensität des Druckes proportional sein, wenn auch individuelle Verhältnisse (Form und Grösse des Augapfels, bzw. Rauminhalt der Orbita, schnelles oder langsames Wachstum der Geschwulst, Höhe des intraokularen Druckes und Resistenz, bzw. Elastizität der Sklera) von Bedeutung sein können.

Der Fall, über den wir im folgenden berichten möchten, ist so einzigartig, dass wir in der uns zur Verfügung stehenden Literatur keinen analogen antreffen konnten. Das kann natürlich nicht bedeuten, dass ähnliche Veränderungen durch Druck eines Tumors auf den Bulbus nicht ab und zu vorkommen. Meist werden sie sich, wenn bei der anatomischen Untersuchung nicht genau darauf geachtet wird, in ihren Anfangsstadien dem Nachweis entziehen, oder durch entzündliche Veränderungen des Bulbus verdeckt und ihre Deutung erschwert, vielleicht auch teilweise auf postmortale Einflüsse bezogen, die in unserm Falle ausgeschlossen sind, da das durch Exenteratio orbitae gewonnene Präparat lebensfrisch untersucht wurde. Der Hauptgrund des seltenen Vorkommens einer derartig hochgradigen Bulbusveränderung beruht aber offenbar darauf, dass verschiedene Momente zusammentreffen müssen, um sie hervorzurufen.

Der 43 jährige Sehriftsetzer W. klagte im Oktober 1907 darüber, dass sein rechtes Auge seit ungefähr 2 Jahren vorgetrieben sei. Es bestand ein Exophthalmus in der optisehen Achse von $3 \mathrm{~mm}$, voller Visus und normaler Hintergrund. Schmerzen und Doppeltsehen waren nicht vorhanden. Es wurde damals ein retrobulbäres Angiom diagnostiziert. Als sich der Patient 6 Jahre später wieder vorstellte, hatte der Exophthalmus auf $10 \mathrm{~mm}$ zugenommen. Der Bulbus war jetzt stark nach oben gedrängt, seine Beweglichkeit nach allen Richtungen, besonders nach unten behindert. Der untere Teil der Bindehaut war stark ehemotisch. Durch das untere Lid liess sich ein anseheinend weicher Tumor durehtasten. Die Hornhaut war diffus getrübt, der Innendruck des Bulbus deutlich vermindert. Der Augenhintergrund war normal, der Visus auf ${ }^{6} / 15$ herabgesetzt.

Da die Zurückdrängbarkeit des Augapfels und das langsame Wachstum der Gesehwulst jedenfalls nicht gegen ein Angiom sprachen, wurde ein Versuch mit der Magnesiumbehandlung nach Payr gemacht, indem zugespitzte Magnesiumstifte durch das untere Lid in den Tumor eingestochen wurden. Unter dieser Behandlung nahm die Protrusion erheblich zu (bis auf $15 \mathrm{~mm}$ ), ebenso die Hornhauttrübung und die Sehstörung. Da sich ausserdem sehr heftige Schmerzen einstellten, machte sich ein operativer Eingriff nötig.

Da sich bei der Operation eine Ausschälung des Tumors mit Erhal- 
tung des Auges als unmöglich erwies und eine profuse Blutung eintrat, wurde die Ausräumung der Orbita vorgenommen. Der Tumor erwies sich bei der anatomischen Untersuchung als ein Fibrosar-

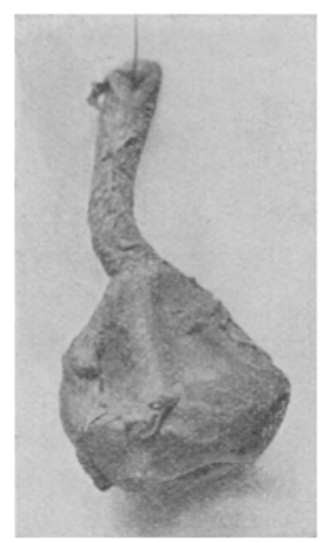

Fig. 1. kom. Er ist in der Dissertation von Lina Marquardt (Leipzig 1914) näher beschrieben worden.

Nach Fixierung in Zenkerscher Lösung und Alkoholhärtung wird der Bulbus vorsichtig vom Orbitaltumor abgelöst, was ohne Scliwierigkeit gelingt. Er sitzt nur mit seiner Unterfläche dem Tumor anf und die Geschwulst hat an keiner Stelle auf die Sklera oder die dem Bulbus anliegenden unteren Augenmuskeln übergegriffen. Die Bulbuswand zeigt sich von unten her an zwei Stellen zu beiden Seiten des Musc. rec. inf. beträchtlich eingedellt. Der untere gerade Augenmuskel ist als vorspringende Leiste zwischen den Dellen deutlich zu erkennen (s. Fig. 1). An sagittal geriehteten medialen Sehnitten ergeben sich folgende Masse: Der Abstand der Papille von zwei korrespondierenden Punkten der Kammerbucht beträgt $19 \mathrm{~mm}$; es beweist dies, dass sich das Verhältnis zwischen

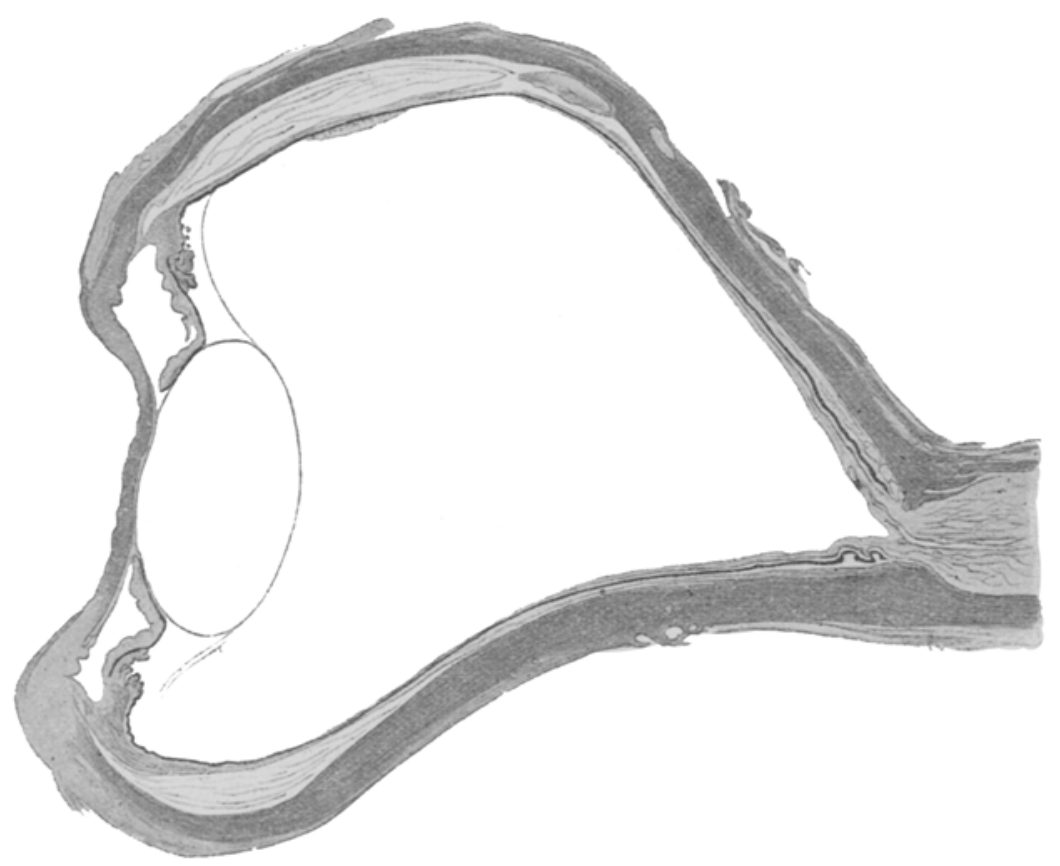

Fig. 2.

vorderem Augenabschnitt und Sehnerveneintrittsstelle nicht geändert hat. Dagegen ist die Form der Bulbuswand, wie sie sich im Sagittalschnitt dar- 
stellt, beträchtlich verändert. Die Sklera bildet keinen Kreisbogen, sondern ist in ihrem Anfangsteil, d. h. vom Canalis opt. sowohl oberhalb als unterhalb des Sehnerveneintritts zu einer geraden Linie gestreckt. Die beiden Geraden schliessen einen Winkel von etwa $60-70^{\circ}$ ein, dessen Spitze in die Mitte der Papille fällt. Durch die ganze Schnittserie lüsst sich dieses Verhalten verfolgen, nur wird der Winkel, je mehr man sich den Seitenteilen nähert, um so spitzer. Es ergibt sich daraus, dass der mittlere Augenabschnitt eine trichterförmigè Gestalt besitzt, der Trichterhals wird gleichsam vom Nervus opticus gebildet. Legt man einen mittleren Sagittalschnitt auf einen analogen eines normalen erwachsenen Auges von gleicher Achsenlänge, so zeigt sich diese trichterförmige Verengerung besonders deutlich. Die Trichterwand ist zum Trichterhals asymmetrisch insofern als die obere Begrenzung des Trichters mit der Achse des Sehnerven einen Winkel von ungefähr $70^{\circ}$ bildet, während die untere Begrenzungsfäche nahezu parallel zur Längsachse des Sehnerven liegt.

Während nun die Sklera in ihrem vorderen Abschnitt in der oberen Bulbushälfte im Sagittalschnitt als eine stark gebogene Kreislinie (mit einem Radius von $8 \mathrm{~mm}$ ) vom Äquator bis zum Hornhautrande verläuft, wobei ihre Konvexität nach aussen gerichtet ist, zeigt sie im nnteren Teile eine Sförmig gekrümmte Kontur, wobei die erste hintere Biegung leicht konvex gegen das Bulbusinnere vorspringt (Radius ungefähr $12 \mathrm{~mm}$ ), während die zweite stärkere Biegung (Radius ungefähr $6 \mathrm{~mm}$ ) ihre Konvexität nach aussen richtet. Durch diese Veränderungen ist der Bulbusinnenraum nach vorn und oben wenig erweitert, nach unten und hinten aber sehr beträchtlich verengt. Der Abstand zwischen zwei korrespondierenden Punkten der inneren Skleralfläche im oberen und unteren Sagittalschnitt beträgt nur $12,5 \mathrm{~mm}$, während er in einem normalen Auge (in gleicher Weise fixiert und gehärtet) $22 \mathrm{~mm}$ beträgt. Die Cornea des, wie die Palpation vor der Enukleation zeigte, hochgradig hypotonischen Bulbus ist stark eingedellt und reicht in der Mitte bis fast zur Pupillarebene und zur vorderen Linsenkapsel, während der Kammerwinkel erweitert, die Iris in der Mitte eingeknickt ist. Durch diese Iriseinknickung und Hornhauteindellung erhält die Kammerbucht einen annähernd rhombischen Querschnitt.

Diese schon makroskopisch deutlich nachweisbaren Formveränderungen des Bulbus konnten natürlich nicht ohne Folgen für das Bulbusinnere sein, besonders für Netzhant, Aderhaut und Sehnerven. Schon bei Betrachtung mit schwacher Vergrösserung fällt auf, dass Netzhaut und Aderhant im Bereich des hinteren Bulbusabschnittes, $d$, h. im Bereich des beschriebenen Skleraltrichters glatt anliegen, während beide zwischen Äquator und Ora serrata flach abgelöst sind. Diese Abhebung kann nicht auf die Einwirkung der Fixierungs- und Härtungsflüssigkeit zurückgeführt werden, denn $a b-$ gesehen davon, dass sich bei fehlerhafter Behandlung nur die Netzhaut abzulösen pflegt, spricht die tadellose Konservierung der Netzhaut and der Nachweis von geronnenem Exsudat zwischen den stark gedehnten Bindegewebsfasern der Suprachorioidea für einen vitalen Prozess.

Die Papille bietet das Bild einer ausgesprochenen Papillitis, ihre Nervenfasern sind beträchtlich aufgelockert und liegen am Papillenrande in etwa $2 \mathrm{~mm}$ breiter Sehicht. Blutungen sind nirgends nachzuweisen, ebenso wenig 
entzüindiche Infltrationen des Papillengewebes. Dagegen sind die Zentralgefässe, besonders die Venen, beträchtlich erweitert. Eine Thrombose lässt sich innerhalb des Venenstammes nicht finden. Der Sehnerv zeigt keine atrophischen Veränderungen (Fürbung nach Wolters-Kultschitzky), de Zwischenscheidenraum ist nicht erweitert, sondern eher verengt. Die adventitiellen Scheiden der Zentralgefässe treten nicht hervor. Verfolgt man die Netzhaut vom Papillenrande nach der Peripherie, so ergibt sich als auffallende Veränderung eine Zusammenschiebung der äusseren Körnerschicht und des Sinnesepithels, die zur Bildung bogenartiger Figuren führt. An der Bildung dieser Bogen ist am stärksten die äussere Körnersehicht und die Stäbchen- und Zapfenschicht beteiligt. Das Sinnesepithel weist im Bereich dieser Bogen deutliche Zerfallserscheinungen auf, besonders die Aussenglieder der Stäbchen und Zapfen sind abgeknickt, stellenweise sogar vollständig geschwunden, während das Sinnesepithel dort, wo die Netzhaut flach der Unterlage anliegt, in keiner Weise verändert ist. Die gegen die Chorioidea gerichtete Konkavität der Bogen zeigt an manchen Stellen einen feinkörnigen Detritus. An den äusseren Körnern lässt sich besonders in den äussersten Lagen, und zwar nur dort, wo Zerfallserscheinungen des Sinnesepithels festzustellen sind. unregelmässige Lagerung, Abblassung und Aufquellung der einzelnen Elemente nachweisen. Die inneren Körner zeigen durchweg normale Struktur, ebenso die grossen Ganglienzellen der inneren Schicht. Das Maximum der Faltenbildung findet sich im unteren Abschnitt des Bulbus etwa $2 \mathrm{~mm}$ vom Papillenrande entfernt. Weiter nach vorn zu sind zwar auch noch Falten anzutreffen, diese sind aber wesentlich weniger steil, und etwa $5 \mathrm{~mm}$ vom Papillenrande entfernt bietet der Netzhantquersehnitt keinerlei Abweichung von der Norm. Im oberen Teil der Netzhaut sind die Falten von vornherein flacher and hören eher anf.

Die Faltenbildung erstreckt sich auch auf die Gegend der Macula. Man sieht hier die schmalen und langen Zapfen an mehreren Stellen von der Unterlage abgelöst und in der Konkavität der Falten Zerfallserseheinungen darbietend, Anch hier beschränkt sich die Faltenbildung vorwiegend auf die äusseren Netzhautschichten, während die stark verbreiterte Nervenfaser- und Ganglienzèllenschicht nur leichte Ausbiegungen erkennen lässt. Die Gefässfüllung der Aderhant, die sich auch auf die Choriocapillaris erstreokt, ist im makularen Bezirk besonders ausgesprochen.

Auch die Aderhaut nimmt an der Zusammenschiebung der Gewebe teil. Man erkennt das am besten aus der bogenförmigen festonartigen Anordnung der Lamina elastica und des Pigmentepithels. Diese Bogen finden sich nur dort, wo die Netzhaut in ihrer änsseren Schicht gefaltet ist. Das Pigmentepithel ist auf der Höhe dieser Bogen teilweise abgelöst und schiebt sich an manchen Stellen unter die abgelöste Netzhautfalte vor. Die Gefässe der Aderhant sind sehr stark erweitert, ihre Lumina enthalten dicht zusammengedrängte rote Blutkörperchen und im Randteile zahlreiche Lymphozyten und Leukozyten. Eine ausgesproehene Infiltration des Aderhautstromas lässt sich dagegen nirgends beobachten. In der Gegend des Äquators zeigen sowohl Netzhant wie Aderhaut wieder normale Verhältnisse. Weiter vorn sind, wie bereits oben erwähnt, beide von der Sklera abgelöst. In diesem abgelösten Teil tritt eine starke Gefässfullung der Aderhaut hervor, und 
man sieht zahlreiche, teilweise zerrissene Fasern der Suprachorioidea, welehe sich zwischen der abgelösten Aderhant und der Sklera ausspannen. Der Ziliarkörper zeigt keine Veränderung, der Kammerwinkel ist weit offen, die Iris ist in ihrem ziliaren Teil den Ziliarfortsätzen angepresst, während sie in ihrem Pupillarteil der vorderen Linsenkapsel anliegt. Das Hornhautepithel ist im Zentrum abgeflacht, teilweise bläschenförmig abgehoben. Die Färbbarkeit der oberflächlichen Hornhautlamellen ist etwas anders als die der tiefen. Nach van Gieson nehmen die vorderen Schichten einen mehr violetten, die hinteren einen karminroten Farbenton an. Das subepitheliale Gewebe ist am Limbus am unteren Teil stark infiltriert, und lässt viele stark

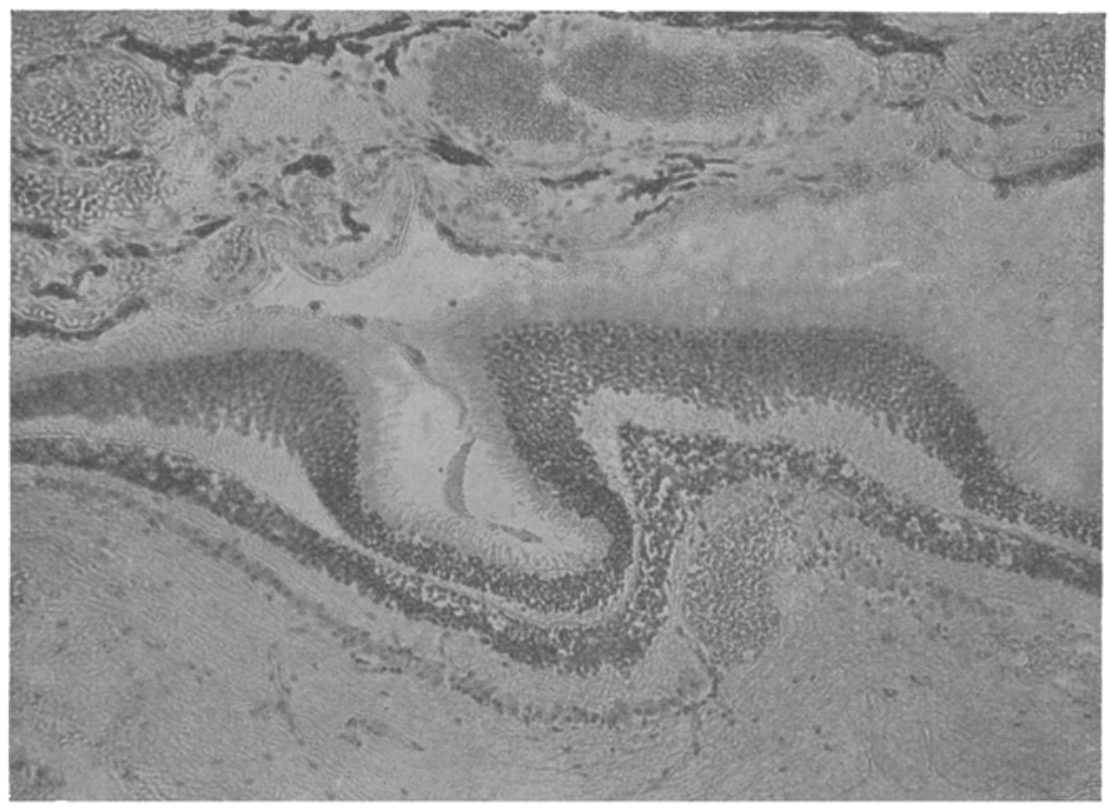

Fig. 3.

gefülte Gefässe hervortreten, während dies am oberen Limbus nicht der Fall ist. Die Descemet ist unverändert. Ein Infiltrat der Cornea ist nicht nachzuweisen.

Was die Deutung unserer Befunde anlangt, so ist zanächst wohl klar, dass sie in erster Linie die mechanischen Verhältnisse ins Auge fassen muss. Der Bulbus wurde im Zustande hochgradiger Protrusion vom Tumor nach oben gedrängt und gegen den oberen Orbitalrand gepresst. Dadurch ist es zu verstehen, dass bei einem Exophthalmus, der für gewöhnlich nicht zu derartigen Veränderungen zu führen pflegt, weil der Augapfel dem Druck nach vorn ausweichen kann, eine so hochgradige Defor- 
mität möglich wurde. Vermutlich kam als weiteres begünstigendes Moment die rapide Zunahme der Protrusion in der letzten Zeit vor der Exenteration hinzu, die als Folge der Magnesiumstiftbehandlung anzusehen ist. Es lässt sich leicht verstehen, dass unter diesen Umständen eine Anpassung des Bulbus an die Druckverhältnisse der Umgebung weniger stattfinden konnte, als bei langsamem Wachstum einer Orbitalgeschwulst. Wesentlich scheint weiter der Umstand, dass der starke Druck des Tumors auf den Bulbus erst erfolgte, nachdem durch einen mässigen, aber langandauernden Druck die Elastizität der Sklera und der intraokulare Druck vermindert war.

Bei tonometrischer Messung exophthalmischer Bulbi haben wir mehrfach auch bei normaler Sehschärfe und unverändertem Hintergrund eine beträchtliche Abnahme der Tension beobachten können. Diese Hypotonie ist wohl in gleicher Weise zu erklären wie diejenige nach Druckverband, d.h. durch Resorption eines Teils der Glaskörperflüssigkeit.

Da es sich nicht um einen diffus infiltrierenden Tumor handelte, sondern um einen solchen, der, aus dichtem Bindegewebe bestehend, von einer Kapsel umgeben und von seiner Nachbarschaft gut abgegrenzt war, fand die Geschwulst, gegen den Bulbus von unten her vordringend, an den Fasern des unteren geraden Augenmuskels einen Widerstand und drängte sich deshalb zu beiden Seiten desselben gegen die Bulbuswand vor.

Der Druck erfolgte offenbar nicht nur von unten nach oben, sondern zugleich von hinten nach vorn, da der Augapfel dem vorderen und oberen Teile des Tumors aufsass. Es musste also nach dem Parallelogramm der Kräfte als Hauptrichtung der Druckwirkung eine schräg von hinten unten nach vorn oben gerichtete Linie in Betracht kommen. So erklärt es sich, dass der hintere Augenabschnitt trichterförmig ausgezogen, der vordere aber von unten her glaskörperwärts eingedellt ist.

Hierdurch wurde der Bulbusinnenraum wesentlich verkleinert. Die Folge musste sein, dass die den Augapfel auskleidenden Membranen in Falten zusammengeschoben wurden, soweit sie nicht elastisch genug waren, sich der veränderten Oberfläche anzupassen. Am deutlichsten ist diese Faltenbildung an den offenbar am wenigsten elastischen äusseren Netzhautschichten nachzuweisen. Dies führte zu der in funktioneller Beziehung wichtigen Konsequenz, dass die Netzhaut sich von. ihrer Unterlage 
hier und da ablöste und durch Zusammenschiebung und gegenseitige Berührung der Falten im Bereiche des Sinnesepithels eine Degeneration der Zapfen- und Stäbchenaussenglieder, zum Teil auch der zugehörigen Körner eintrat. Da die Faltenbildung sich in unserem Falle bis zur Macula verfolgen liess, würde eine beträchtliche Störung auch des zentralen Visus erklärt werden. Leider liess sich hierüber ebensowenig ein sicheres Urteil gewinnen wie über das ophthalmoskopische Aussehen dieser kleinen Netzhautfalten, da die Hornhaut getrübt und eingesenkt, die Spiegeluntersuchung unmöglich und bei der starken Verdrängung des Bulbus nach oben eine genaue Sehprüfung ausgeschlossen war. Nach der anatomischen Beschaffenheit der Netzhaut ist aber eine Verminderung der Sehschärfe durch die Faltenverschiebung und ihre Folgen sicher anzunehmen.

Eine weitere Folge des Druckes des Tumors auf den Bulbus bildet die hochgradige venöse Stauung, die sowohl im Sehnerven als in der Netzhaut und Aderhaut nachzuweisen war. Dass diese Stauung durch Zerrung oder partielle Verlegung der hinteren Abflusswege (Vena centralis retinae, Venae vorticosae) veranlasst wurde, ist kaum zu bezweifeln. Die Stauung im Gefässgebiet der Vena centralis kann jedoch auch auf die Stauungspapille bezogen werden, wenn man nicht, wozu wohl mehr Grund vorliegt, da die Zwischenscheidenräume und die perivaskulären Lymphspalten im Opticus nicht erweitert waren, umgekehrt die venöse Stase als Ursache der Papillenschwellung ansprechen will.

Bemerkenswert ist weiterhin die Abhebung der Aderhaut im vorderen Bulbusabschnitt. Wir können uns gut vorstellen, dass bei einem Druck, der in schräger Richtung auf den Bulbus von unten her ausgeübt wurde, eine Zerrung der Aderhaut bewirkt wurde, die zur Abhebung von der Sklera führte, um so mehr, da die Elastizität der Chorioidea durch die pralle Füllung der Gefässe, wenn nicht aufgehoben, so doch stark vermindert war.

Wie hätten sich nun, wenn es gelungen wäre, den Tumor mit Erhaltung des Bulbus zu entfernen, die Verhältnisse im Auge weiter gestaltet? Hierüber sind nur Vermutungen möglich. Man kann sich wohl denken, dass beim Nachlassen des Tumordrucks und bei noch gut funktionierenden Ziliarfortsätzen der normale Bulbusinnendruck sich nach und nach wiederhergestellt haben würde und der Augapfel seine normale Form wiedergewonnen hätte. Dabei wären dann die Netzhautfalten, soweit sie noch nicht 
412 A. Birch-Hirschfeld und C. Siegfried, Zur Kenntnis d. Veränderungen usw verklebt waren (was bei der Untersuchung noch nicht der Fall war), geglättet worden. Es ist aber natürlich auch möglich, dass sich aus dem Bilde, das wir bei der Untersuchung fanden, eine Netzbautabhebung entwickelt hätte, war doch durch die Faltenbildung der Zusammenhang zwischen Aderhaut und Netzhaut bereits an vielen Stellen gelockert.

Wir möchten vermuten, dass ähnliche Veränderungen des Bulbus, wie wir sie in unserm Falle antrafen, wenn auch vielleicht in geringerem Grade und relativ selten, d. h. nur, wenn analoge mechanische Verhältnisse in Frage kommen, ab und zu nachzuweisen sind. Möchte diese Mitteilung zu ihrem genaueren Studium Anlass geben, da sie nicht nur in anatomischer, sondern auch in funktioneller Hinsicht unser Interesse beanspruchen dürfen. 